\title{
Intervención con jóvenes mexicanos en conflicto ante la ley desde la psicología de la comunidad
}

Ángel Corchado-Vargas ${ }^{\star}$

* Doctor en Educación. Profesor, Universidad Nacional Autónoma de México, México.

Correo electrónico:

angel.corchado@campus.iztacala.unam.mx

Recibido: 7 de abril del 2016

Aprobado: 11 de agosto del 2016

Cómo citar este artículo: Corchado-Vargas, A. (2016). Intervención con jóvenes mexicanos en conflicto ante la ley desde la psicología de la comunidad. Pensando Psicología, 12(20), 85-95. doi: http://dx.doi.org/10.16925/ pe.v12i20.1566

\section{Resumen}

Propósito: describir las actividades que se han realizado en el contexto de la aplicación del modelo de la psicología de la comunidad con jóvenes mexicanos (llamados "menores infractores") que, por diversas circunstancias, han sido remitidos a las denominadas preceptorías juveniles, ubicadas en el estado de México, en el área metropolitana de la República Mexicana. Tema: el conocimiento de las actividades que se han generado con menores infractores permitirá, prospectivamente, diseñar estrategias de intervención cada vez más pertinentes con la población mencionada desde la psicología social comunitaria. Desarrollo: se enuncian algunas consideraciones sobre la manera como se forma a los estudiantes de la carrera de Psicología de la Facultad de Estudios Superiores (FES) Iztacala, UNAM, en el ámbito de la investigación, a fin de enunciar la manera como se puede realizar una intervención eficaz con menores infractores mexiquenses. Se enumeran las distintas intervenciones que se han realizado en las preceptorías juveniles, así como la metodología empleada, poniendo de manifiesto los avances de dicha intervención y las propuestas de mejora en el trabajo que llevan a cabo los alumnos de la carrera de Psicología de la Universidad Nacional Autónoma de México, fEs Iztacala. Conclusiones: se espera que este modelo sirva para la formación de estudiantes en escenarios similares en Latinoamérica, considerando la pertinencia ética y el compromiso social que se tiene dentro del ámbito social, así como la dinámica deductiva e inductiva que conlleva el proceso de investigación-intervención.

Palabras clave: compromiso social, menores infractores, preceptorías juveniles, psicología de la comunidad. 


\title{
Intervention with Young Mexicans in Conflict with the Law Based on Community Psychology
}

\begin{abstract}
Purpose: To describe the activities that have been carried out in the context of the application of the community psychology model to Mexican youth (called "Juvenile Offenders") who, for various reasons, have been referred to the so-called juvenile detention centers, located in the state of Mexico, in the metropolitan area of Mexico. Topic: Awareness of the activities that have been carried out with juvenile offenders will prospectively allow to design increasingly relevant intervention strategies for the population concerned based on community social psychology. Development: At first, some considerations on how psychology students from the School of Higher Studies (FEs by its Spanish acronym) Iztacala, UNAM, are trained in the field of research are discussed and then the manner in which an effective intervention with Mexican juvenile offenders can be made is presented. The various interventions that have been made in juvenile detention centers are listed, as well as the methodology used, showing the progress of such intervention and proposals for improvement in the work carried out by psychology students from the Universidad Nacional Autónoma de México, fes Iztacala. Conclusions: It is expected that this model will serve to train students in similar scenarios in Latin America, considering ethical relevance and social commitment to the social sphere, as well as deductive and inductive dynamics involved in the research-intervention process.
\end{abstract}

Keywords: social commitment, juvenile offenders, juvenile detention centers, community psychology.

\section{Intervenção com jovens mexicanos no conflito perante a lei desde a psicologia da comunidade}

\section{Resumo}

Escopo: descrever as atividades que foram realizadas no contexto da aplicação do modelo da psicologia comunitária com jovens mexicanos (chamados de "Menores Infratores") que, por diversos motivos, têm sido encaminhados às chamadas preceptorias juvenis, localizadas no estado de México, na zona metropolitana da República Mexicana. Tema: o conhecimento das atividades que se tem gerado com menores infratores irá permitir, prospectivamente, projetar estratégias de intervenção cada vez mais pertinentes com a população citada desde a psicologia social comunitária. Desenvolvimento: na primeira instancia, enunciam-se algumas considerações sobre a maneira em que os estudantes da carreira de Psicologia da Faculdade de Estudos Superiores (FES) Iztacala, UNAM, são formados no âmbito da investigação, para depois enunciar a forma como pode ser feita uma intervenção eficaz com menores infratores mexicanos. São enumeradas as diversas intervenções realizadas nas preceptorias juvenis, bem como metodologia empregada, evidenciando os avanços dessa intervenção e as propostas de melhoramento no trabalho que está sendo executado por parte dos alunos da carreira de Psicologia da Universidade Nacional Autónoma de México, FES Iztacala. Conclusões: espera-se que este modelo sirva para a formação de estudantes em cenários similares na América Latina, considerando a pertinência ética e o engajamento social que se tem dentro do âmbito social, bem como a dinâmica dedutiva e indutiva que envolve o processo de investigação - intervenção.

Palavras chave: engajamento social, menores infratores, preceptorias juvenis, psicologia da comunidade. 


\section{Introducción}

Una de las principales características de la Universidad Nacional Autónoma de México se refiere al carácter indagatorio de los fenómenos de tipo social. Sería prácticamente imposible concebir nuestra alma máter desvinculada del proceso de investigación. Todos nosotros, que somos parte del cuerpo universitario en todos los niveles - desde los alumnos, hasta docentes e investigadores- sabemos de antemano que aquel que no investiga no es parte del proceso de construcción del hecho educativo en la universidad.

La carrera de Psicología en la Facultad de Estudios Superiores Iztacala se ha ocupado del estudio de los individuos a nivel social y a nivel individual. Para ello, desde que se fundamentó el plan de estudios, este proceso de abordaje se concibió en dos vertientes o niveles: el unidimensional y el multidimensional.

El primer nivel es empleado desde el momento en el cual el estudiante de Psicología se confronta con el hecho educativo de la investigación básica. Desde una perspectiva individual y social, el alumno emplea como herramientas la segmentación, la cuantificación, la medición y el control experimental. En sus primeros pasos, el alumno-investigador se aboca al establecimiento de la relación causa-efecto, y a la observación medible y cuantificable de los fenómenos sin ocuparse, según algunas perspectivas, de los procesos que los produjeron.

Más adelante, conforme el alumno va adquiriendo conocimientos, herramientas y habilidades más complejos para los procesos de investigación - e intervención-, puede ser capaz de emplear el nivel multidimensional, el cual está definido como una totalidad estructurada, ya que estudia procesos y no únicamente productos. El nivel multidimensional social le permite al investigador llevar a cabo un análisis dinámico y cualitativo de los procesos de la conducta de los individuos, entendiendo como análisis cualitativo una descripción del "cómo" y el "por qué", más que de un "qué" del proceso o fenómeno que se aborda.

Otro aspecto que se toma en cuenta en el nivel multidimensional es el carácter relacional de un individuo con el grupo al que pertenece, y para indagar esta relación se utilizan instrumentos de investigación social tales como entrevistas, cuestionarios, encuestas, escalas de actitud y tests sociométricos, que como técnicas de observación indirecta son de mucha utilidad, aunados a las técnicas de observación directa tales como técnicas grupales, tareas dirigidas, registros anecdóticos, diarios de campo, observación participante, historias de vida y todo lo que lleve consigo una observación in vivo del problema que se aborda.

En el contexto de la trayectoria académica de nuestros estudiantes, como parte del plan de estudios de la carrera de Psicología, nos encontramos ante un reto por demás relevante que confronta a nuestros alumnos con una realidad: el módulo de Psicología Social Aplicada. El abordaje de grupos en las instituciones, la interacción entre personas en diferentes escenarios, el estudio de estos y la inmersión del psicólogo social en dichos contextos son temáticas necesarias y con una fuerza importante para cumplir con el perfil de egreso de la licenciatura:

El Psicólogo que se forma en la Facultad de Estudios Superiores Iztacala es capaz de identificar, valorar, proponer alternativas de prevención y solución para diversas situaciones derivadas de la interacción entre individuos, grupos, instituciones y comunidades para el desarrollo del ser humano y la sociedad. Además, cuenta con las siguientes habilidades: Detecta, evalúa, diagnostica, planea, diseña, rehabilita, investiga y modifica comportamientos o interacciones disfuncionales, diseña, desarrolla planes y programas de capacitación (Iztacala.unam.mx, s. f.).

El perfil de egreso de nuestra licenciatura exige que los alumnos cada vez sean más críticos, con mayores capacidades de análisis, interiorización y desprofesionalización ${ }^{1}$ hacia los fenómenos conductuales y sociales; por lo tanto, cuando hablamos de un trabajo con grupos - en el caso particular de este trabajo, el que se refiere a los jóvenes en conflicto ante la ley-, sustentado en una firme teoría y en una práctica supervisada, retroalimentada y reorientada en sus procesos, no tendremos más que reconocer que el psicólogo egresado de nuestra facultad sea, hoy por hoy, la persona que por perfil profesional y por capacidad académica y de competencia académica tenga

1 La desprofesionalización es concebida como una "dimensión” de las actividades profesionales. Las actividades profesionales prevén dos dimensiones bien definidas. La primera, la intervención directa del psicólogo en la solución de problemas concretos o en el desarrollo de soluciones nuevas. La segunda es la actividad del psicólogo mediada por y paraprofesionales y no-profesionales (Vargas-Mendoza, 2011). 
toda la autoridad para el abordaje, la interpretación y la intervención en psicología social aplicada. No podríamos concebir la construcción social de nuestro entorno sin mediar un proceso de análisis de los procesos sociales, entre ellos el de los grupos de jóvenes que por diversas circunstancias han estado involucrados en un acto ilícito, sea como protagonistas o como copartícipes.

Todos los elementos expuestos denotan la relevancia que el módulo aplicado en el área de psicología social tiene para la formación de nuestros estudiantes, asimilando así la concordancia entre el perfil de egreso del psicólogo de Iztacala y el proceso formativo del que son sujetos y agentes en el último año escolar.

\section{Desarrollo del contenido temático}

El presente tema escrito tiene como objetivo explicar la forma como se ha llevado a cabo una intervención con jóvenes en conflicto ante la ley, partiendo del modelo de la psicología social aplicada en el ámbito comunitario, también denominada psicología de la comunidad.

Para iniciar, es importante delimitar a quiénes nos referimos cuando estamos hablando de jóvenes. Las edades cronológicas del desarrollo humano están más determinadas por procesos sociales y culturales que por situaciones cronológicas o fisiológicas. Algunos autores hablan de edades más o menos constantes y frecuentes para delimitar la juventud. Papalia y Wendkos (2002) especifican la existencia de una etapa de juventud, la cual se presenta posteriormente a la adolescencia, dividiéndola en juventud temprana, media y tardía. En contraste, y de acuerdo con enfoques más actuales, Kail y Cavanaugh (2011) realzan la importancia de los elementos sociales (tales como los ritos de iniciación, el despertar de la sexualidad, el gregarismo entre iguales, etc.) para definir la etapa del ciclo vital que esté viviendo el sujeto, más allá de la edad cronológica. Así pues, se puede encontrar, de acuerdo con estas características, cómo la línea divisoria entre la adolescencia y la juventud es por demás difusa. Dado lo anterior, se considera pertinente justificar la ubicación de la población que nos ocupa en el presente tema más por el contexto social y cultural en el que se desarrollan que por una edad determinada por los años de vida.

Entenderemos, pues, que un joven es aquella persona que deja de ser niño por el convencionalismo social-cultural, y que comienza a experimentar conductas, actitudes, ideas, pensamientos, necesidades, intereses y afectos cualitativamente distintos a los que experimentaba cuando era niño, siendo algunos de ellos considerados de riesgo social y jurídico (Izquierdo, 2012).

En México, particularmente, la población joven es una de las más representativas a nivel nacional. Se habla que más de la mitad de la población es aún joven, y en cuanto a los determinantes jurídicos, es necesario recurrir a la Ley para el Tratamiento de Menores Infractores para el Distrito Federal en Materia Común y para toda la República en Materia Federal, cuya función, tal como lo refiere su articulado, es la de reglamentar la protección de los derechos de los menores, así como en su adaptación social. La conducta del menor infractor se encuentra tipificada en las leyes penales federales y del Distrito Federal. Dicha ley establece criterios claros de protección al menor, los cuales consideran que el menor infractor no merece castigo, sino protección y asistencia jurídica especiales, por lo cual debe ser sometido a un régimen especial que pueda denominarse "derecho de los menores", quedando, por lo tanto, fuera del Derecho Penal (Cruz y Cruz, 2010). Este criterio es el que ha adoptado preferentemente la legislación mexicana. En apoyo a la idea anterior, Ruiz (2000) menciona que los menores infractores son todas aquellas personas que no rebasan la mayoría de edad y que han cometido hechos o acciones violatorias de reglamentos o de leyes penales, independientemente de que sean o no registrados por las autoridades responsables o de que los hechos sean ocasionales o habituales, los cuales que no pueden ser tomados como normales en el proceso evolutivo o social. En el caso de los jóvenes-adolescentes que han cometido alguna falta que va de lo leve a lo grave ${ }^{2}$, se han diseñado dos escenarios previstos para alojar a esta población: los consejos tutelares y las preceptorías juveniles. El consejo tutelar tiene la finalidad de promover la readaptación social de los menores de 18 años que hayan cometido algún ilícito o que tengan cierta inclinación a causarse daño personal, a la familia y a la sociedad por medio del estudio de la personalidad, la aplicación de medidas correctivas y de protección, y la vigilancia

\footnotetext{
2 Las faltas leves son, por ejemplo, algún hurto menor en los centros escolares o el vecindario, alguna falta administrativa como las pintas o grafitis, mientras que se consideran graves el desacato a la autoridad, conductas obscenas, complicidad en actos vandálicos, robo a casa o habitación, asociación en delitos contra la salud y homicidios.
} 
del tratamiento (Tocaven, 1999). Las preceptorías juveniles son instancias en las que los menores infractores son sometidos a un tratamiento y a la reincorporación social por medio del externamiento ${ }^{3}$. Estas tienen como propósito llevar a cabo acciones y actividades que contribuyan a la reintegración de adolescentes en conflicto con la ley, o que presenten alguna conducta antisocial, mejorando de esta forma su calidad de vida. Se logra a través de la participación del cuerpo técnico interdisciplinario, cuyas principales funciones son la de estructurar planes y programas individuales, familiares y sociales, con la finalidad de darles a conocer las alternativas que existen y dar solución a la problemática presente, previniendo además la reincidencia y los malos hábitos.

El psicólogo social forma parte de este cuerpo técnico, ya que él es el encargado de contribuir en la preparación de las estrategias de intervención con los jóvenes-adolescentes y de ejecutar algunas de ellas. Sus funciones están encaminadas en el ámbito de la prevención y del tratamiento, así como de la dotación de herramientas que promuevan la conciencia personal y social y el logro de la no reincidencia.

En cuanto a las instalaciones, las Preceptorías Regionales de Reintegración Social cuentan con espacios destinados para aulas escolares, canchas deportivas, áreas verdes, talleres de capacitación, salón de usos múltiples, etc. (DGPYRS, GEM, 2009). Dichas instalaciones son explotadas de manera suficiente para poder llevar a cabo el trabajo de intervención con los jóvenes-adolescentes.

Ahora bien, antes de comentar y describir algunas de las actividades que el psicólogo social puede llevar a cabo con esta población, resulta necesario explicar algunas de las causas que llevan a los jóvenes-adolescentes a presentarse en las instancias de acompañamiento, sean los consejos tutelares o las preceptorías juveniles. Si bien las causas que se mencionarán a continuación no son todas, al menos sí son las más frecuentes a las que nos hemos enfrentado a lo largo de estos casi diez años de trabajar con esta población.

\footnotetext{
3 Se entiende por externamiento el hecho de que los menores infractores que han cometido el ilícito no tienen que estar en resguardo o internamiento en la institución, pero deberán reportarse por medio de la presencia física a tantas actividades como la preceptoría lo requiera.
}

\section{La familia}

Hurlock (1979) menciona que las relaciones entre los individuos raras veces se mantienen estáticas. La gente cambia y de igual modo varían sus relaciones con los demás. En particular, nos ocuparemos de la familia. Menciona que los altercados familiares por la búsqueda de la emancipación familiar llegan a ser más frecuentes conforme el joven-adolescente crece, afirmando que "el costo por la independencia del joven llega a ser muy alto más cuando el grado de libertad concedido al hijo por parte de los padres no es el mismo grado de independencia que pretende el adolescente" (Hurlock, 1979, p. 441). Cuando el adolescente desea más de lo que los padres le han concedido, comienzan los altercados familiares. Por otra parte, para Fitzgerald et al. (1997), hogares desechos, extrema pobreza, crueldad, abuso, fracasos en la escuela y alcoholismo dentro del hogar son algunas de las principales razones que han llevado a los jóvenes a "huir de la casa", y esto se convierte en otra causal para que el adolescente delinca y repercuta en la sociedad.

\section{La delincuencia juvenil per se}

Son todos aquellos actos ilegales cometidos por menores de edad, los cuales se considera que tienen gran relación con variables familiares y con las llamadas "bandas" y "pandillas"; sin embargo, es probable que estos actos tengan un estrecho vínculo con la interacción de otra clase de variables (psicológicas y sociológicas). Con respecto a los actos ilegales de los menores infractores de las preceptorías juveniles donde se lleva a cabo la intervención, se encuentran con frecuencia: ultraje, robo, robo con violencia, robo a mano armada, abuso sexual, violencia sexual, agresiones, intento de homicidio y homicidio; además, de acuerdo con datos de la preceptoría juvenil de Tlalnepantla, en el 2006, alrededor de un 90\% de estos jóvenes perteneció a alguna pandilla antes de ser sujeto a proceso legal, y cerca del $70 \%$ proviene de familias disfuncionales.

4 Si bien huir de la casa no es en sí un delito, esta situación se encuentra dentro del perfil observado en los expedientes de los menores que cometieron algún delito y que acuden a las preceptorías juveniles de Tlalnepantla, Atizapán, Naucalpan y Cuautitlán, México. No es generalizable a todos los casos, pero en la realidad próxima se halla presente. 


\section{El entorno violento}

Entendido como el daño físico voluntario que se infringe a otra persona, o el daño o la destrucción de la propiedad ajena. Es frecuente que a las preceptorías juveniles donde se ha intervenido sean remitidos de manera permanente jóvenes-adolescentes que han golpeado o lesionado a compañeros o familiares, o bien han dañado bienes inmuebles del vecindario.

\section{Consumo de drogas y alcohol}

Entendido como la ingesta de sustancias prohibidas, si bien este es un evento de origen multifactorial, se han podido observar hechos (esencialmente de índole social) que de manera concomitante impactan la tendencia al consumo de drogas y alcohol: familias en conflicto, frustración por los pocos logros obtenidos en la escuela, fracaso escolar, influencia de amistades o pareja y problemas en el noviazgo. Entre el 2005 y el 2008, en las preceptorías juveniles de Atizapán y Tlalnepantla, más del $60 \%$ de los jóvenes-adolescentes que acudieron manifestó haber consumido algún tipo de droga (principalmente activo y marihuana); sin embargo, muchos no se consideraron adictos, ya que solo - dijeron- la habían probado un par de ocasiones. Estos datos fueron reportados por los adolescentes de manera directa dentro de las actividades desarrolladas en la preceptoría.

\section{Cambios cognitivos: en el constructo de sus ideas ante la vida y conformación como personas}

Entre estos se encuentra el fracaso escolar, el cual deriva en deserción. En las preceptorías juveniles de Cuautitlán, entre el 2010 y el 2011, así como en Tlalnepantla, desde el 2004, se han mencionado como agentes que propician el abandono de los estudios no solo el fracaso ya mencionado, sino también la necesidad económica, la falta de interés y el embarazo precoz (Encuesta Nacional de la Juventud, 2000). Cuando hay necesidad de dinero, no siempre el trabajo formal o informal es la alternativa; recurren también al delito. La falta de habilidades sociales lleva consigo la consecución de un delito, ya que muchos jóvenes que no tienen desarrolladas habilidades proactivas y de toma de decisiones hacen lo más fácil y sencillo (y una de las conductas más fáciles es la delictiva). Por otra parte, una de las situaciones comunes y constantes a las que se enfrenta el adolescente en la actualidad es la mencionada por Marín de Ordaz (1997), la cual consiste en clichés comerciales que son ya también eventos que lastiman a los menores de edad y buscan obligarlos a adquirir, en algunas ocasiones de manera desleal, mercancías innecesarias para satisfacer necesidades artificiales.

Resultaría necio pretender poner a los jóvenes-adolescentes que delinquen como víctimas de las circunstancias. Sería una forma fácil de abordar la problemática y focalizar la responsabilidad en el entorno o medio que los rodea. Resultaría también injusto, ya que estaríamos socavando la capacidad que tienen los jóvenes de tomar las riendas de sus vidas, y a la vez ensalzaríamos el poder de los adultos sobre ellas. Partiendo de estos breves ejemplos de situaciones a las que nos hemos enfrentado en la práctica, resultará idóneo replantear el nivel de responsabilidad que le corresponde potencializar al joven-adolescente. Así mismo, promover que las familias, cuidadores y funcionarios del Gobierno que se encargan del cuidado y la reinserción social de nuestros menores infractores también participen en el logro de su acomodo a la norma, a la vida cordial y a la sana convivencia.

Partiendo de esta idea, reflexionemos en torno a las palabras que Rivera (2004, p. 47) plantea al respecto:

Las emociones [...] se manifiestan hacia la sociedad en actitudes y hacia sí mismo en sentimientos, dolores y enfermedades, por tal es necesario que el joven aprenda a poner atención en su cuerpo; es decir, estar en contacto con sus emociones y consigo mismo y una forma de hacerlo es que el muchacho aprecie y viva con los sentidos y enseñarle que todo aquello que se proponga lo haga con voluntad, perseverancia y amor en todos sus actos.

Las palabras del autor ponen de manifiesto, en lo implícito, la meta que tiene la psicología social comunitaria al intervenir con la persona: promover su libertad, autogestión y responsabilidad, libre de paternalismos, proteccionismos y dependencias. Desde esta perspectiva, no hay intención de apoyo en la comunidad, en los grupos y las instituciones si el profesional de la psicología no logra la autonomía en los seres humanos con los que trabaja (Buelga et al., 2009; Tovar, 2001). En el caso de la intervención con jóvenes-adolescentes que han cometido alguna falta y que han tenido problemas con la ley, no será realmente 
efectivo el trabajo si se genera una subordinación hacia la figura del profesional de la psicología.

Quienes llevamos a cabo la intervención con esta población tan peculiar, habremos de sumergirnos en el mundo subjetivo de quien pertenece a esa realidad, para comprenderlo, acompañarlo y así promover el cambio (Gómez del Campo, 2011).

Hasta este punto, se han descrito algunas de las definiciones y el encuadre de la problemática que nos ocupa con los jóvenes que presentan problemas ante la ley. Ahora será pertinente delimitar la forma en que se puede intervenir con esta población desde una perspectiva de la psicología social. Como se ha puesto de manifiesto anteriormente, es la vertiente de la psicología social comunitaria la que pretende promover, gracias a que los resultados previos han sido favorables.

Se dividirá la descripción de la intervención en tres ámbitos.

\section{El ¿qué?}

Mediante este se rescatará un análisis histórico de las intervenciones que se han hecho con esta población, bajo la supervisión de la práctica de quien presenta este tema escrito con el nombre que se le otorgó a la intervención.

\section{El ¿cómo?}

Por medio del cual se explicará lo que se a hecho en las distintas intervenciones.

\section{El ¿para qué?}

Sin esta explicación la razón de ser de una práctica de psicología social no tendría fundamento. Por un lado, lo que se logró a nivel de impacto con la comunidad $\mathrm{y}$, por otro lado, lo que se logró a nivel de investigación, considerando que la psicología social aplicada no solo persigue fines de intervención con las personas, grupos e instituciones, sino también parte de realidades concretas y fomenta la investigación.

Las figuras 2-10 presentan de manera esquemática la cronología de las acciones que se han llevado a cabo dentro de las preceptorías juveniles del Estado de México, especificando los tres ámbitos de la intervención.

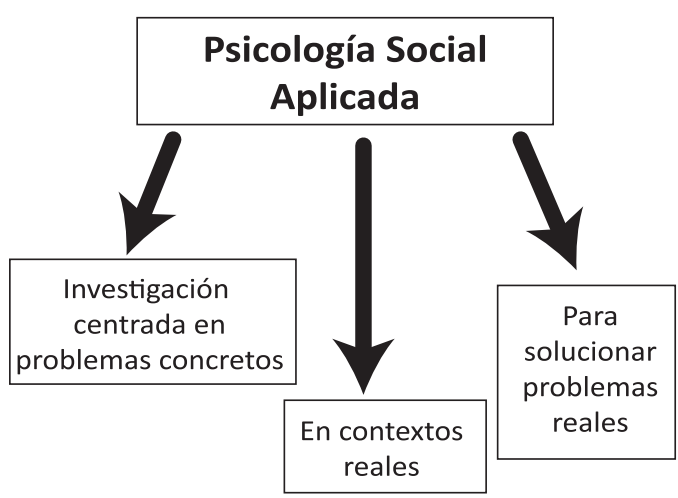

Figura 1. Fines que persigue la Psicología Social Aplicada. Elaboración propia.

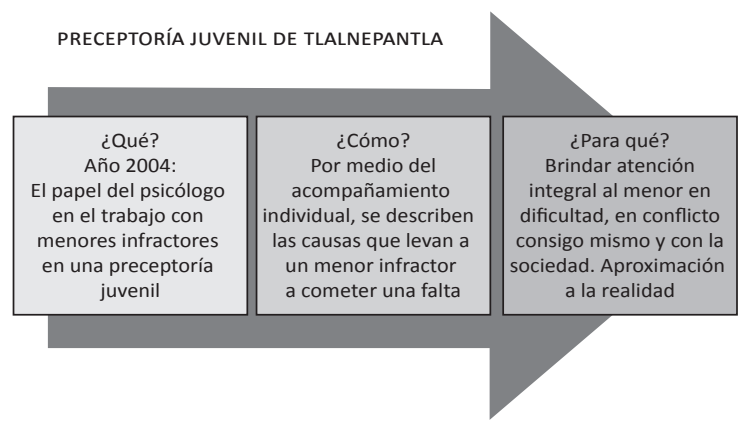

Figura 2. Actividades en el 2004. Elaboración propia.

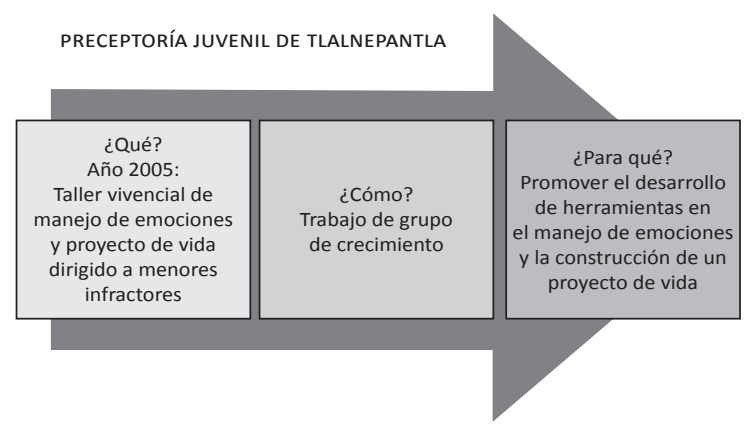

Figura 3. Actividades en el 2005. Elaboración propia. 


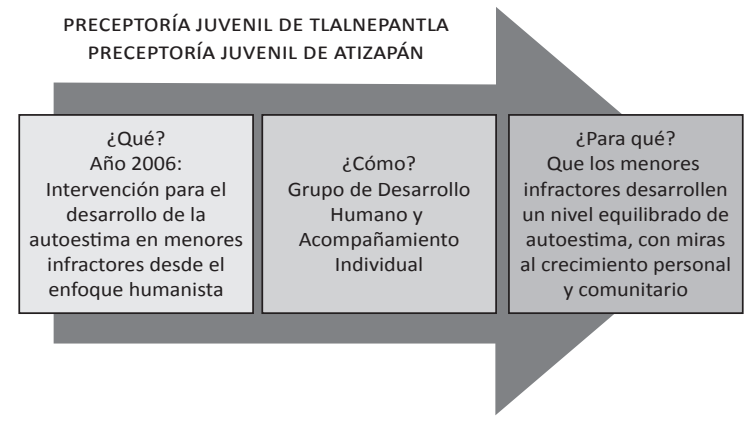

Figura 4. Actividades en el 2006. Elaboración propia.

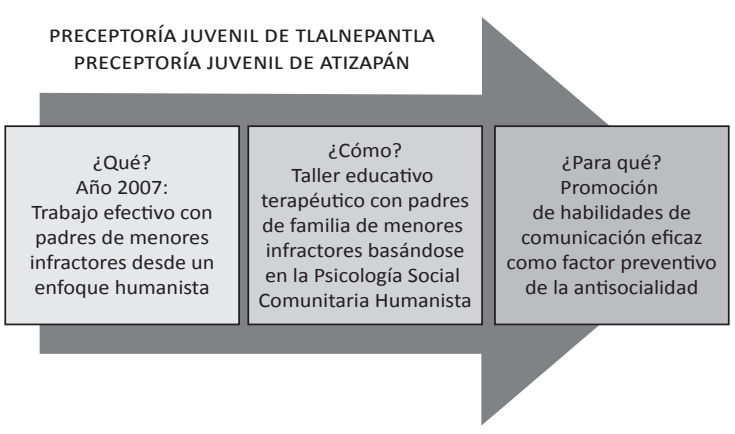

Figura 5. Actividades en el 2007. Elaboración propia.

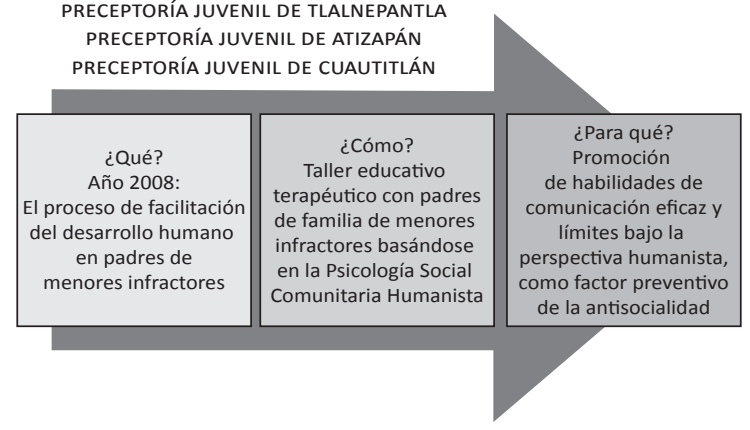

Figura 6. Actividades en el 2008. Elaboración propia.

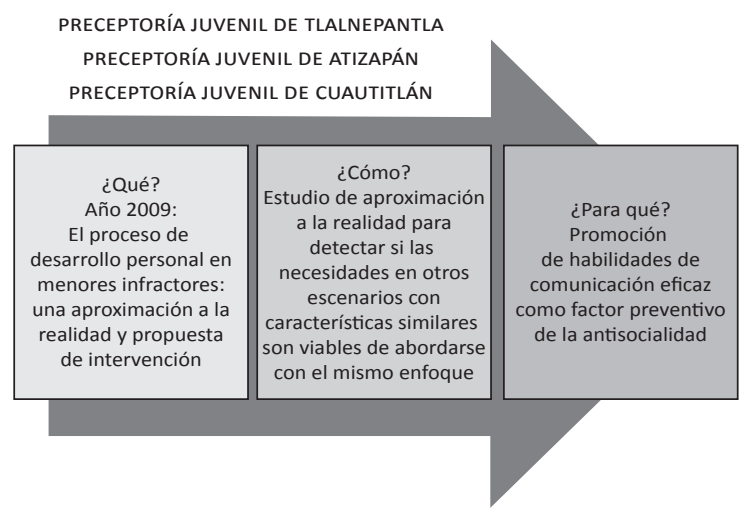

Figura 7. Actividades en el 2009. Elaboración propia.

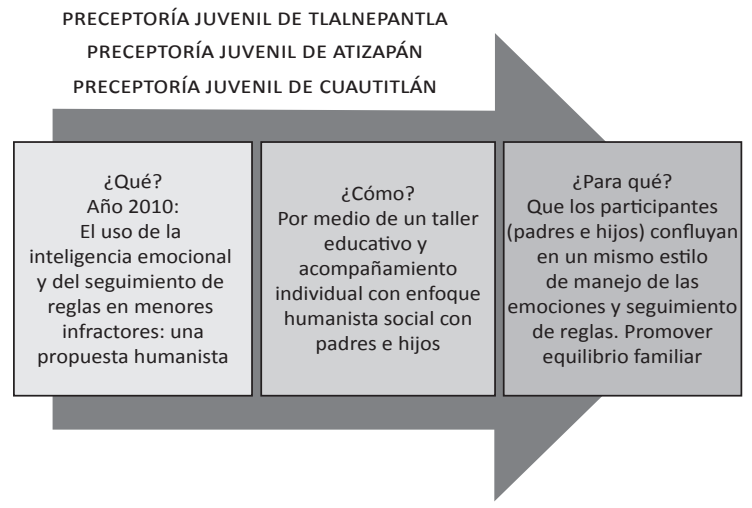

Figura 8. Actividades en el 2010. Elaboración propia.

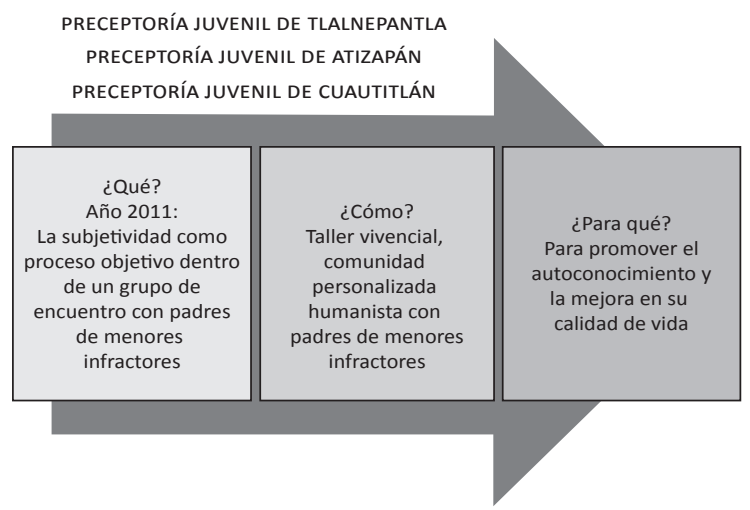

Figura 9. Actividades en el 2011. Elaboración propia. 


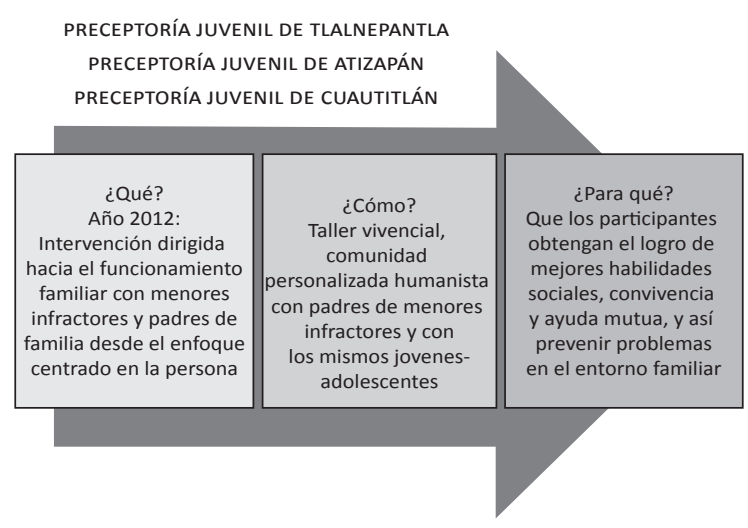

Figura 10. Actividades en el 2012. Elaboración propia.

\section{Algunas consideraciones}

Dada la envergadura de las intervenciones antes descritas, los estudiantes han podido reportar sus resultados de la intervención oportunamente al concluir el ciclo escolar, pero incluso han deseado permanecer en la institución por más tiempo. Una bondad o ganancia ha redundado en que los contraten para seguir formándose en el área de atención a menores infractores, o incluso han perfeccionado sus trabajos de práctica y los han convertido en documentos de titulación.

En ciertos años se ha interrumpido la intervención en algunas preceptorías juveniles, debido al cambio de políticas y autoridades internas en cada una de las instancias en las que se ha trabajado. También ha influido el hecho de que la saturación de alumnos que acuden a hacer servicio social (no precisamente de nuestra facultad sino de otras instituciones), entorpecen los trabajos que se han venido realizando. A partir de este ciclo (2013), ya no estamos en prácticas en la preceptoría juvenil de Tlalnepantla.

\section{Metodología}

En todos los casos se ha empleado la metodología cualitativa, dada la importancia que se le brinda a la cualidad de los eventos observados sobre la cantidad de estos. Dentro de esta metodología se encuentra el método fenomenológico, el cual permite comprender la realidad a partir de la visión particular del ser humano a quien se le pregunta; es decir, se estudian los fenómenos a partir de la experiencia subjetiva, tal como lo experimenta, vive y percibe (Martínez, 2001). La fenomenología tiene sus raíces en la filosofía de Husserl y su objetivo central es el campo de los fenómenos, tal como son vividos y experimentados por los individuos. Es en sí la investigación sistemática de la subjetividad; es una investigación a través de la cual se busca la comprensión de cuatro existenciales básicos: el espacio vivido, el cuerpo vivido, el tiempo vivido y las relaciones humanas vividas. Es por ello que se dice que los existencialistas han sido el puente utilizado hacia el conocimiento de las vivencias (Álvarez-Gayou, 2007; Merlino, 2008). La fenomenología pretende estudiar aquellos eventos que, desde las percepciones, sentimientos y acciones de los actores sociales, aparecen como significativos; es decir, pretende comprender los fenómenos sociales desde la propia perspectiva de los actores (Murcia y Jaramillo, 2003). En este sentido, el trabajo que se ha llevado a cabo con jóvenes que enfrentan problemáticas ante la ley puede ser explicado de manera óptima y clara por medio de la fenomenología.

Álvarez-Gayou (2007) explica también que por medio de la fenomenografía podemos centrar la manera como son experimentados diferentes fenómenos, y las formas de percibirlos, a fin de que se conozcan y se desarrollen habilidades sobre ellos ${ }^{5}$. Su objetivo es encontrar las variaciones de acuerdo con los diversos factores del fenómeno. Aunque se pueden utilizar diversos tipos de datos en este paradigma interpretativo, el más común es la entrevista individual y el trabajo personalizado.

Así pues, la fenomenología como encuadre y la fenomenografía como técnica permitirán que se pueda entender de manera cabal la realidad que viven, en este caso, los jóvenes-adolescentes que han infringido alguna norma, así como las personas cercanas a ellos.

Martínez (2001) menciona que el método fenomenológico comprende tres etapas, tal como se muestran en la figura 11.

La utilización de la fenomenología a lo largo del tiempo ha permitido que se pueda descubrir la realidad de quienes la viven desde las entrañas. Es un método holístico, ya que los elementos se relacionan con las otras partes a través de múltiples interacciones, en el cual cada uno va adquiriendo una función que solo se explica en la conjunción de las partes con el todo.

\section{Conclusiones}

La intervención con la población de jóvenes que han tenido problemas con la ley no puede reducirse a la

Ad hoc con los objetivos que se han planteado en las diversas intervenciones. 


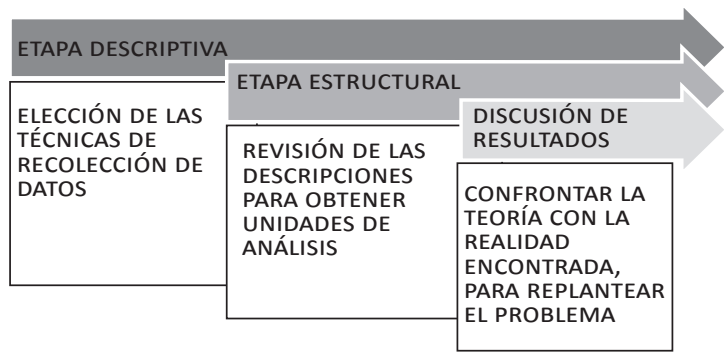

Figura 11. Etapas del método fenomenológico según Martínez (2001). Elaboración propia.

descripción, también se enfoca en la intervención y en la formulación de estrategias que permitan que el trabajo sea cada vez más vívido, realista y acorde con los tiempos actuales. Resulta por demás necesaria la revisión bibliográfica y empírica acerca del trabajo que se ha llevado a cabo con este tipo de poblaciones, pero deberá realizarse también un concienzudo análisis acerca de la pertinencia de los textos con la realidad a la cual nos enfrentamos. Por esto, es importante que los estudiantes que acuden a hacer su servicio social en las instancias en las que se atiende a menores infractores documenten de manera detallada cuáles son las vertientes más socorridas en la realidad de los jóvenes. Es necesario generar líneas de intervención e investigación ad hoc con la cultura, el entorno social y la realidad inmediata que rodea a nuestros personajes.

Sería penoso utilizar la intervención social con este grupo reduciéndonos a describir lo que pasó y cómo ocurrió. Es necesario formular propuestas de mejora en el ámbito del sistema legal, social, familiar y, obviamente, psicológico, a fin de promover el desarrollo comunitario de quienes experimentan y vivencian el delito como ejecutores y como receptores. El trabajo de investigación-intervención habrá de moverse en dos direcciones: en la deducción, para así partir de ideas generales, principios teóricos e investigaciones previas; y movernos dinámicamente al plano de la inducción: para promover conjeturas en las que se considere que, si se promueven condiciones similares bajo las mismas circunstancias de promoción del desarrollo personal de los jóvenes en problemas legales, se podrá lograr una mejora en el ámbito social y comunitario.

Finalmente, en el ámbito de la ética profesional, habremos de ser cautelosos en el trabajo que se lleva a cabo con una población tan vulnerable como esta. No olvidar que, como psicólogos sociales, estamos para promover el desarrollo comunitario, fundamentado en la libertad y la autonomía, en el desapego a la dependencia que ellos (los jóvenes y sus familias) puedan depositar en nosotros. No obstante, el hecho de realizar una intervención e investigación no nos convierte en espectadores del fenómeno. Somos parte de él mismo; nos involucramos y pertenecemos al fenómeno. Es parte de nosotros. Solo así asumiremos nuestro verdadero compromiso social como coadyuvantes en el desarrollo de nuestra sociedad y de la disciplina psicológica.

\section{Referencias}

Álvarez-Gayou, J. (2007). Cómo hacer investigación cualitativa. Fundamentos y metodología. México: Paidós.

Buelga, S., Musitu, G., Vera, A., Ávila, M. y Arango, C. (2009). Psicología social comunitaria. Ciudad de México: Trillas.

Cruz y Cruz, E. (2010). El concepto de menores infractores. Ciudad de México: unam. Recuperado de http:// www.juridicas.unam.mx/publica/librev/rev/posder/ cont $/ 5 / \mathrm{cnt} / \mathrm{cnt} 17 . \mathrm{pdf}$

Dirección General de Prevención y Reinserción Social, Gobierno del Estado de México - DGPYRs, GEM. (2009). Recuperado de http://qacontent. edomex.gob.mx/ dgprevencion/inicio/index.htm

Fitzgerald, H., McKinney, J. y Strommen, E. (1997). Psicología del desarrollo. Ciudad de México: Manual Moderno.

Gómez del Campo, J. (2011). Psicología de la comunidad. Ciudad de México: Plaza y Valdés.

Hurlock, E. (1979). Psicología de la adolescencia. México: Paidós.

Iztacala.unam.mx. (s. f.). Carrera de Psicología. Recuperado de http://www.iztacala.unam.mx/nuevo_ingreso/? page_id $=1124$

Izquierdo, M. (2012). Menores infractores en México. Revista sobre Infancia y Adolescencia, 3, 24-32.

Kail, R. y Cavanaugh, J. (2011). Desarrollo humano. Una perspectiva del ciclo vital. Ciudad de México: Cengage Learning.

Marín de Ordaz, G. (1997). Los menores infractores. Ciudad de México: Olmos.

Martínez, M. (2001). La psicología humanista: fundamentación epistemológica, estructura y método. Ciudad de México: Trillas. 
Merlino, A. (2008). Investigación cualitativa en ciencias sociales. Temas, problemas y aplicaciones. Ciudad de México: Cengage Learning.

Murcia, N. y Jaramillo L. (2003). Investigación cualitativa. Bogotá: Kinesis.

Papalia, D. y Wendkos, S. (2002). Desarrollo humano. Ciudad de México: McGraw-Hill.

Rivera, M. (2004). El adolescente, su cuerpo y la sociedad moderna. Ciudad de México: CEAPAC.

Ruiz, G. (2000). Menores infractores. Una pedagogía especializada. Ciudad de México: Ediciones Castillo.
Tocaven, R. (1999). Menores infractores. Ciudad de México: Edicol.

Tovar, M. (2001). Psicología social comunitaria. Una alternativa teórico-metodológica. Ciudad de México: Plaza y Valdés.

Vargas-Mendoza, J. (2011). El proceso de desprofesionalización de la psicología: apuntes para Iztacala. Notas: Boletín Electrónico de Investigación de la Asociación Oaxaqueña de Psicología A.C., 7(1). 29-34. Recuperado de http://goo.gl/OsJFYh 